\title{
Desigualdad de género en las actividades de prevención de riesgos laborales
}

\section{Gender inequality in occupational risk prevention}

\section{María Gayoso Doldan', Shirley Rodríguez Tupayachi ${ }^{2}, \mathrm{M}^{a}{ }^{a}$ Carmen Fernández Felipe ${ }^{3}, \mathrm{M}^{a}{ }^{a}$ Luisa de la Cruz Cantos $^{4}$}

1. Mutua Universal. Madrid. España

2. Servicio de Prevención. Hospital Universitario Fundación Alcorcón. Madrid. España

3. Servicio de Prevención. Hospital Universitario de la Princesa. Madrid. España

4. Servicio de Prevención. Hospital Universitario de Getafe. Madrid. España

Recibido: 19-12-14

Aceptado: 23-12-14

\section{Correspondencia}

María Gayoso Doldan

solergayoso@gmail.com

Este artículo se realiza dentro del Programa de Investigación MIR de la Escuela Nacional de Medicina del Trabajo, del Instituto de Salud Carlos III. Madrid. España

Resumen

Introducción: Los objetivos de este estudio, para comprobar la existencia de una posible desigualdad de géneros en relación a las actividades preventivas, consisten en conocer la frecuencia con la que la mujer tiene acceso a la vigilancia de salud, en qué medida recibe formación e información, la frecuencia con que se realiza la evaluación de los riesgos para su salud en el puesto de trabajo, que aspectos del puesto de trabajos se estudian además de la evaluación de riesgos tanto en los aspectos organizativos, como de los aspectos psicosociales.

Material y métodos: Estudio epidemiológico observacional transversal, de fuente secundaria, a partir de los datos de la «VII Encuesta Nacional de Condiciones de Trabajo, realizada por INSHT en el 2011. Para la determinación de la desigualdad entre géneros se utilizó el test de Chi cuadrado para las variables cualitativas y el T-test para muestras independientes para variables cuantitativas. Para el control de posibles interacciones o modificaciones del efecto se realizó un análisis de regresión logística binaria, la significación de los modelos se contrastó mediante la pruebas de Hosmer y Lemeshow.

Resultados: El 56.30\% de mujeres frente a 43.70\% de hombres, afirman que no se les ofreció la posibilidad de realizarse el reconocimiento médico al 52,80\% de mujeres que afirman que si se les ofreció el reconocimiento médico no se lo llegaron a hacer. A un $51.70 \%$ de mujeres frente a un $48.30 \%$ de hombres no se les realizó la evaluación de riesgos en el puesto de trabajo. El $56.10 \%$ de mujeres frente a un $43.90 \%$ de hombres afirman considerarse mal informados sobre los riesgos de su puesto de trabajo. En el análisis de regresión los factores más relacionados con una menor accesibilidad a actividades de prevención de riesgos laborales fueron: el ser mujer, la ausencia del delegado de prevención, el contrato temporal, y ser trabajador extranjero. Concluimos que el ser mujer trabajadora supone un menor acceso a la gestión preventiva.

Med Segur Trab (Internet) 2015; 61 (238) 4-17

Palabras claves: Género, desigualdad, medio laboral, trabajo. 


\section{Abstract}

Objectives: In order to test the possible gender inequality in labour prevention, we need to know how often women have access to health surveillance, which is the scope of the training and information received by women, the frequency with which the assessment of health risks in the workplace.

Methods: Cross-sectional epidemiological study of secondary source data from the "Seventh National Working Conditions Survey, conducted by INSHT in 2011.

Results: $56.30 \%$ women compared to $43.70 \%$ of men say they were not offered the medical examination in the last 12 months, while the $52.80 \%$ of women, who were offered medical examination, did not attend. Risk assessment in workplace during the last 12 months was not performed to $51.70 \%$ of women versus $48.30 \%$ men. $56.10 \%$ of women versus $43.90 \%$ of men claim to be misinformed about the risks of their job. We got constant risk factors: being female, the absence of prevention delegate, temporary contract, and being foreign worker.

Med Segur Trab (Internet) 2015; 61 (238) 4-17

Keywords: Gender, inequality, work environment, work. 


\section{INTRODUCCIÓN}

En la IV Conferencia sobre la Mujer celebrada Pekín se estableció el principio de transversalidad o perspectiva de la igualdad de género incorporándolo al marco jurídico europeo $^{1}$.

En 2006 La Unión Europea propone acciones a través del Pacto europeo para la igualdad de género y su promoción fuera de la Unión Europea ${ }^{2}$. Nace en España la Ley Orgánica 3/2007, de 22 de marzo, a objeto de hacer cumplir el derecho de igualdad mediante la eliminación de la discriminación de la mujer ${ }^{3}$.

En 2012 la OIT publica el programa «Igualdad de Género y Trabajo Decente», y como parte de éste, el trabajo productivo y decente en condiciones de libertad, seguridad, equidad, dignidad humana, Justicia e igualdad para hombres y mujeres incluido los jóvenes ${ }^{4}$.

A pesar de la amplia normativa en el ámbito nacional e internacional que vincula las acciones de las entidades públicas y privadas con la igualdad de género para el pleno acceso al mercado laboral, así como el derecho al empleo remunerado, educación, formación vocacional, seguridad y promoción en el trabajo. El 10\% de los ingresos por el empleo en el mundo, los recibe la mujer, pero realizan dos tercios del trabajo mundial no remunerado $^{5}$.

En España existen desigualdades de género tanto en las condiciones de empleo, medio ambiente de trabajo, como en los problemas de salud relacionados con el trabajo y conciliación de la vida laboral y familiar ${ }^{6}$.

Con referencia a la prevención de riesgos laborales en un estudio realizado por José Luis Llorca Rubio, Pedro R. Gil-Monte se concluye que el género es una variable que puede generar una discriminación negativa hacia la mujer. Y que los riesgos laborales y los problemas de salud asociados deberían ser considerados un problema de salud pública $^{7}$.

Roquelaure Y, Ha C, Rouillon C, señala, la incapacidad de las instituciones para conocer la situación real de las y los trabajadores y por tanto, la imposibilidad de emprender acciones que promuevan adecuadamente la salud de hombres y mujeres en dicho medio ${ }^{8}$.

En la identificación de los riesgos en el ámbito de la salud laboral se considera a hombres y mujeres como iguales en factores fisiológicos, antropométricos, hormonales y sociales cuando realmente no lo son y se les considera diferentes en aspectos como capacidad de trabajo, aptitudes técnicas, dotes de organización incluso en la posibilidad de enfermar de patologías tradicionalmente «masculinas» como las cardiovasculares 9.

Para la Salud Pública los problemas que afectan a las mujeres son básicamente los relacionados con la salud sexual reproductiva, los otros problemas son sesgados con apreciaciones estereotipadas, sobre la salud mental, falta de ajuste al trabajo, factores hormonales o subjetividad, M. Zimmermann ${ }^{10}$.

La incorporación de la perspectiva de género en las acciones de los Servicios de Prevención contribuye a detectar y corregir los sesgos que discriminan a la mujer en las actividades preventivas y de vigilancia de la salud ${ }^{11}$.

Las políticas de Seguridad y Salud Ocupacional, dirigidas a la promoción de la salud de la mujer, deben para su elaboración tener en cuenta todas las funciones que desempeña: como amas de casa, como madres y como trabajadoras ${ }^{12}$.

Dados los antecedentes que existen en el tema nos planteamos comprobar la hipótesis de que existe una desigualdad de géneros en relación a las actividades preventivas. Siendo los objetivos de este estudio, conocer la frecuencia con la que la mujer tiene acceso a la vigilancia de salud, en qué medida recibe formación e información, la frecuencia con que se realiza la evaluación de los riesgos para su salud en el puesto de 
trabajo, que aspectos del puesto de trabajos se estudian además de la evaluación de riesgos tanto en los aspectos organizativos, como de los aspectos psicosociales.

Así, la importancia de considerar las diferencias entre hombres y mujeres reconociendo los distintos factores que pueden influir en los riesgos a los que se enfrentan unos y otros en su trabajo se traducen en adoptar estrategias de prevención adecuadas bajo la perspectiva de género ${ }^{13}$.

\section{METODOLOGÍA}

A objeto de proceder a la verificación de la hipótesis planteada acerca de la desigualdad de género que hay en cuanto a las actividades preventivas y a la consecución de los objetivos formulados, se ha diseñado un estudio observacional transversal, de fuente secundaria, a partir de los datos de la «VII Encuesta Nacional de Condiciones de Trabajo" (VII ENCT), realizada por el Instituto Nacional de Seguridad e Higiene en el Trabajo (INSHT) en el año 2011.

El trabajo de campo de la VII E. N. C. T. se realizó entre octubre 2011 y febrero de 2012. La encuesta cubre el ámbito geográfico de todo el territorio nacional a excepción de Ceuta y Melilla. Se ha realizado sobre una muestra representativa a nivel nacional de 8.892 trabajadores activos a partir de 16 años, y de todas las actividades económicas, mediante entrevista personal en el domicilio del trabajador.

Esta encuesta consta de 62 preguntas distribuidas en 14 apartados. De las cuales elegimos para este estudio, aquellas vinculadas a la con las actividades preventivas (tabla 1).

Tabla 1. Variables de la VII ENCT incluidas en el estudio

\begin{tabular}{ll}
\hline \multirow{2}{*}{ SEMOGRÁFICAS } & Sexo \\
\cline { 2 - 2 } Edad & Nacionalidad \\
\hline $\begin{array}{l}\text { En los últimos doce meses, ¿se le ofreció, por parte de su empresa } \\
\text { o centro donde realiza su trabajo, la posibilidad de pasar un } \\
\text { reconocimiento médico? }\end{array}$ \\
\cline { 2 - 2 } En los últimos doce meses, ¿se ha realizado una evaluación o algún \\
estudio de los riesgos para su salud o seguridad en su puesto de \\
trabajo? \\
$\begin{array}{l}\text { En relación con los riesgos para su salud y seguridad relacionados } \\
\text { con su trabajo, ¿en qué medida diría Vd. que está bien informado? }\end{array}$ \\
$\begin{array}{l}\text { En los dos últimos años, ¿ha recibido formación o información } \\
\text { sobre los riesgos para su salud y seguridad relacionados con su } \\
\text { trabajo? }\end{array}$ \\
$\begin{array}{l}\text { Evaluación de riesgo en los aspectos organizativos: horarios, carga } \\
\text { de trabajo, tareas desempeñadas }\end{array}$ \\
$\begin{array}{l}\text { Evaluación de riesgo en los aspectos psicosociales: relaciones } \\
\text { interpersonales, supervisión de los jefes, participación de } \\
\text { trabajadores, promoción, violencia en el trabajo }\end{array}$ \\
\begin{tabular}{l} 
¿Cómo es su contrato? \\
\hline ¿Qué tipo de jornada tiene en su trabajo, completa o parcial? \\
(horario de mañana o tarde)
\end{tabular} \\
$\begin{array}{l}\text { Sector en el que trabaja, CNAE } \\
\text { OCUPACIONALES }\end{array}$ \\
\hline
\end{tabular}

Fueron excluidos los registros que no tenían información completa en las preguntas o indicadores objeto de análisis. 
Se realizó el análisis descriptivo calculando las frecuencias absolutas y relativas para las variables cualitativas, la media y parámetros de dispersión para la edad.

Para la determinación de la desigualdad entre géneros se utilizó el test de Chi cuadrado para las variables cualitativas y el T-test para muestras independientes para variables cuantitativas. La significación se consideró con $\mathrm{p}<0.05$.

Para el control de posibles interacciones o modificaciones del efecto se realizó un análisis de regresión logística binaria, en la que las variables independientes polinómicas se incluyeron como variables Dummy. La significación de los modelos se contrastó mediante la pruebas de Hosmer y Lemeshow.

Los valores originales de las variables relacionadas a la actividad preventiva se dicotomizarón, considerándose en las nuevas variables como «exposición o situación de riesgo" el valor 1 y "no riesgo" el valor 0 .

"La Carga mental» se generó como una variable nueva dicotómica, considerándola positiva en el caso de respuesta afirmativa a cualquiera de las siguientes variables originales: "Alto nivel de atención", "Ritmo elevado» o "Mucho trabajo". Para el riesgo de "Carga ergonómica" se generó una variable dicotómica considerándola positiva en el caso de respuesta afirmativa a cualquiera de las siguientes variables originales: "Adoptar posturas dolorosas o fatigantes", "Levantar o mover cargas pesadas", "Levantar o mover personas", "Aplicar fuerzas importantes", "Repetir los mismos movimientos de manos o brazos".

Los valores de las variables originales (tipo likert de 5 categorías) se dicotomizaron, considerando como exposición las categorías "a menudo" y "siempre o casi siempre» $\mathrm{y}$ como no exposición las categorías "raramente" $\mathrm{y}$ "casi nunca o nunca", el valor medio de la escala se consideró como valor perdido.

En la (Tabla 2) se muestran las variables independientes incluidas en los modelos de regresión. Como variables dependientes se utilizaron: "Tener acceso al reconocimiento médico» "Recibir Formación e información sobre los riesgos inherentes a su puesto de trabajo", "Si se realiza evaluación de riesgos en el puesto de trabajo", "Si se evalúan los riesgos en los aspectos organizativos y en los aspectos psicosociales».

Tabla 2. Variables independientes incluidas en el estudio

\begin{tabular}{ll}
\hline VARIABLE INDEPENDIENTE & CATEGORÍA DE REFERENCIA \\
\hline Plantilla del centro de trabajo & 1 a 10 \\
\hline Nivel de estudios & Sin estudios \\
\hline Exposición a carga mental & Sin carga mental \\
\hline Nacionalidad & Español \\
\hline Existencia de delegado de prevención & Con delegado \\
\hline Tipo de contrato & Fijo \\
\hline Trabajar en el sector agrario & No agrícola \\
\hline Trabajar en el sector industria & No industria \\
\hline Trabajar en el sector de la construcción & No construcción \\
\hline Exposición a carga ergonómica & Sin carga Ergonómica \\
\hline Inhalar contaminantes químicos & No inhala químicos \\
\hline Manipular compuestos químicos & No manipula químicos \\
\hline Estar expuesto a ruido elevado & No expuesto a ruido \\
\hline Trabajar en el sector servicios & No servicios \\
\hline Tipo de jornada & Tiempo completo \\
\hline Sexo & Varón \\
\hline
\end{tabular}




\section{RESULTADOS}

En la muestra representativa a nivel nacional de 8892 trabajadores (obtenida de la VII ENCT) observamos en el (Gráfico 1) que aunque hay mayor cantidad de hombres que de mujeres entre los entrevistados la diferencia no es relevante.

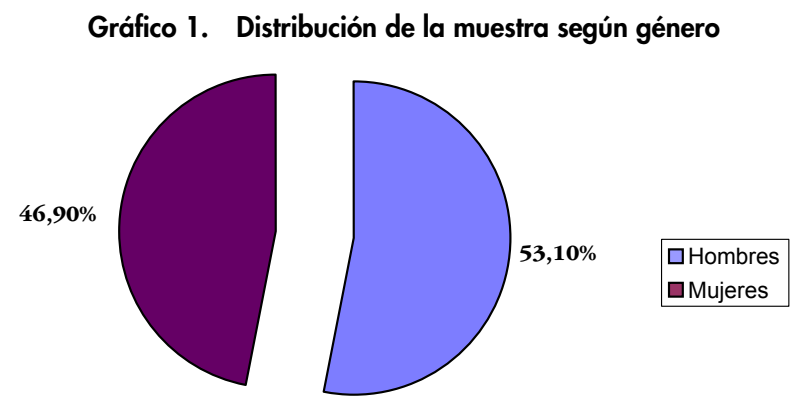

En la distribución de la población según edad y género (Tabla 3), observamos que hay un incremento similar en ambos géneros a partir de los 25 años que se corresponde con el inicio de la vida laboral y aunque la mayoría de hombres y mujeres se concentran en los grupos de y edad de 35 a 44 años y 45 a 54 años se puede apreciar un descenso progresivo y sostenido de la población laboral femenina.

Tabla 3. Distribución de la población según edad y género

\begin{tabular}{|c|c|c|c|c|c|}
\hline \multirow{2}{*}{\multicolumn{2}{|c|}{ EDAD POR GÉNERO }} & \multicolumn{2}{|c|}{ Hombre } & \multicolumn{2}{|c|}{ Mujer } \\
\hline & & $\mathbf{N}^{o}{ }^{\circ}$ & $\%$ & $\mathbf{N}^{\circ}$ & $\%$ \\
\hline \multirow{6}{*}{$\begin{array}{l}\text { EDAD (agrupando } \\
\text { los de más edad) }\end{array}$} & 16 a 24 años & 153 & 50,2 & 152 & 49,8 \\
\hline & 25 a 34 años & 977 & 51,0 & 940 & 49,0 \\
\hline & 35 a 44 años & 1.532 & 52,8 & 1.370 & 47,2 \\
\hline & 45 a 54 años & 1.363 & 54,8 & 1.126 & 45,2 \\
\hline & 55 y más años & 694 & 54,8 & 573 & 45,2 \\
\hline & No contesta & 4 & 36,4 & 7 & 63,6 \\
\hline \multirow[t]{2}{*}{ Total } & & 4.723 & 53,1 & 4.168 & 46,9 \\
\hline & & & 100,0 & & 100,0 \\
\hline
\end{tabular}

En lo referente a la nacionalidad (Gráfico 2) observamos que mientras que en el peso de la emigración europea se encuentra en el hombre, en otras nacionalidades el mayor peso específico se concentra en la mujer.

Gráfico 2. Distribución de la muestra según la nacionalidad por género

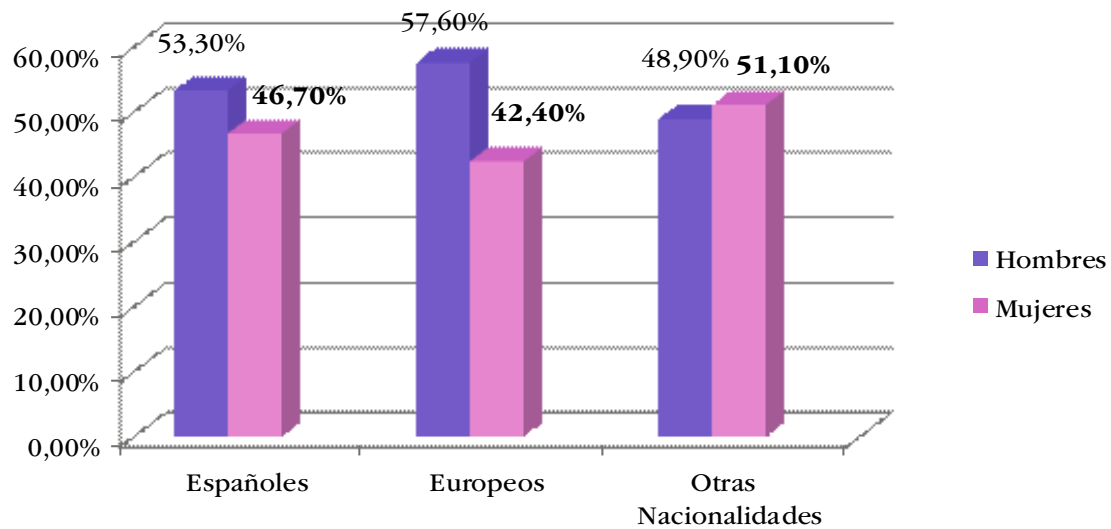


En el tipo de contrato (Tabla 4) se evidencia el predominio estadísticamente significativo $(\mathrm{p}=0,000)$ de la mujer en los contratos: fijo discontinuo, contrato eventual, de formación, e interino, así como en el contrato temporal a través de una ETT.

Tabla 4. Distribución de la población según género y tipo de contrato

\begin{tabular}{|c|c|c|c|c|c|}
\hline \multirow{2}{*}{ Tipo de contrato } & \multicolumn{2}{|c|}{ Hombre } & \multicolumn{2}{|c|}{ Mujer } & \multirow{2}{*}{$\mathbf{P} \leq$} \\
\hline & $\mathbf{N} .^{o}$ & $\%$ & $\mathbf{N .}^{\circ}$ & $\%$ & \\
\hline Indefinido & 2.744 & 52,6 & 2.476 & 47,4 & \multirow{9}{*}{0,000} \\
\hline Fijo discontinuo & 192 & 46,5 & 221 & 53,5 & \\
\hline Por obra o servicio & 481 & 52,5 & 435 & 47,5 & \\
\hline Eventual por circunstancias de la producción & 134 & 49,3 & 138 & 50,7 & \\
\hline Interino & 49 & 32,5 & 102 & 67,5 & \\
\hline De formación & 9 & 45,0 & 11 & 55,0 & \\
\hline En prácticas & 9 & 56,3 & 7 & 43,8 & \\
\hline Temporal a través de una ETT & 18 & 48,6 & 19 & 51,4 & \\
\hline Total & & 100,0 & & 100,0 & \\
\hline
\end{tabular}

El análisis del tipo de horario laboral (Tabla 5) muestra un claro predominio estadísticamente significativo $(\mathrm{p}=0,000)$ de la mujer con respecto al hombre en las jornadas fija de mañana y fija de tarde.

Tabla 5. Distribución de la población según género y tipo de horario laboral

\begin{tabular}{|c|c|c|c|c|c|}
\hline \multirow{2}{*}{ Tipo de horario laboral } & \multicolumn{2}{|c|}{ Hombre } & \multicolumn{2}{|c|}{ Mujer } & \multirow{2}{*}{$\mathbf{P} \leq$} \\
\hline & $\mathbf{N} .^{\circ}$ & $\%$ & $\mathbf{N} .^{\circ}$ & $\%$ & \\
\hline Jornada Partida: Mañana y tarde & 2.215 & 62,0 & 1.358 & 38,0 & \\
\hline Jornada Continua: Fijo mañana & 1.047 & 41,1 & 1.501 & 58,9 & \\
\hline Jornada Continua: Fijo tarde & 111 & 27,4 & 294 & 38,0 & \\
\hline Jornada Continua: Fijo Noche & 106 & 68,8 & 48 & 31,2 & \\
\hline Horario en equipos rotativos(turnos): Mañana/ Tarde & 688 & 53,2 & 606 & 46,8 & 0,000 \\
\hline Horario en equipos rotativos(turnos): Mañana/ Tarde/Noche & 399 & 62,0 & 245 & 38,0 & \\
\hline Horario en equipos rotativos(turnos): Otro tipo de turno & 30 & 75,0 & 10 & 25,0 & \\
\hline Otra variable irregular. Especificar & & 55,4 & & 44,6 & \\
\hline Total & & 100,0 & & 100,0 & \\
\hline
\end{tabular}

En cuanto a la categoría profesional (Tabla 6) se aprecia una diferencia significativa $(\mathrm{p}=0,000)$ donde la mujer tiene un mayor peso en el segmento de empleados, e igualmente observamos que a medida que los puestos disminuyen en categoría va aumentando la presencia de la mujer.

Tabla 6. Distribución del tipo de puesto de trabajo según género

\begin{tabular}{lrrrr}
\hline \multirow{2}{*}{\multicolumn{1}{c}{ Tipo de puesto de trabajo }} & \multicolumn{2}{c}{ Hombre } & \multicolumn{2}{c}{ Mujer } \\
\cline { 2 - 5 } & \multicolumn{1}{c}{. $^{{ }^{\circ}}$} & \multicolumn{1}{c}{$\%$} & N. $^{\text {o }}$ & $\%$ \\
\hline Empleado (con jefes y sin subordinados) & 3.006 & 47,9 & 3.265 & 52,1 \\
\hline Encargado, jefe de taller o de oficina, capataz o similar & 367 & 70,0 & 157 & 30,0 \\
\hline Mando intermedio. Director de pequeña empresa, departamento o sucursal & 373 & 63,9 & 211 & 36,1 \\
\hline Director de empresa grande o media & 47 & 77,0 & 14 & 23,0 \\
\hline Autónomo (sin jefes y sin subordinados) & 656 & 63,6 & 375 & 36,4 \\
\hline
\end{tabular}


En lo referente tipo de jornada (Gráfico 3) se observa un predominio significativo $(\mathrm{p}=0,05)$ de la mujer en la jornada a tiempo parcial.

También la jornada parcial, es superior en la mujer respecto al hombre. Mientras que el tipo de jornada completa es la más frecuente en el hombre.

Gráfico 3. Distribución de la población según género y tipo de jornada laboral

(*) $\mathrm{P}<0.05$

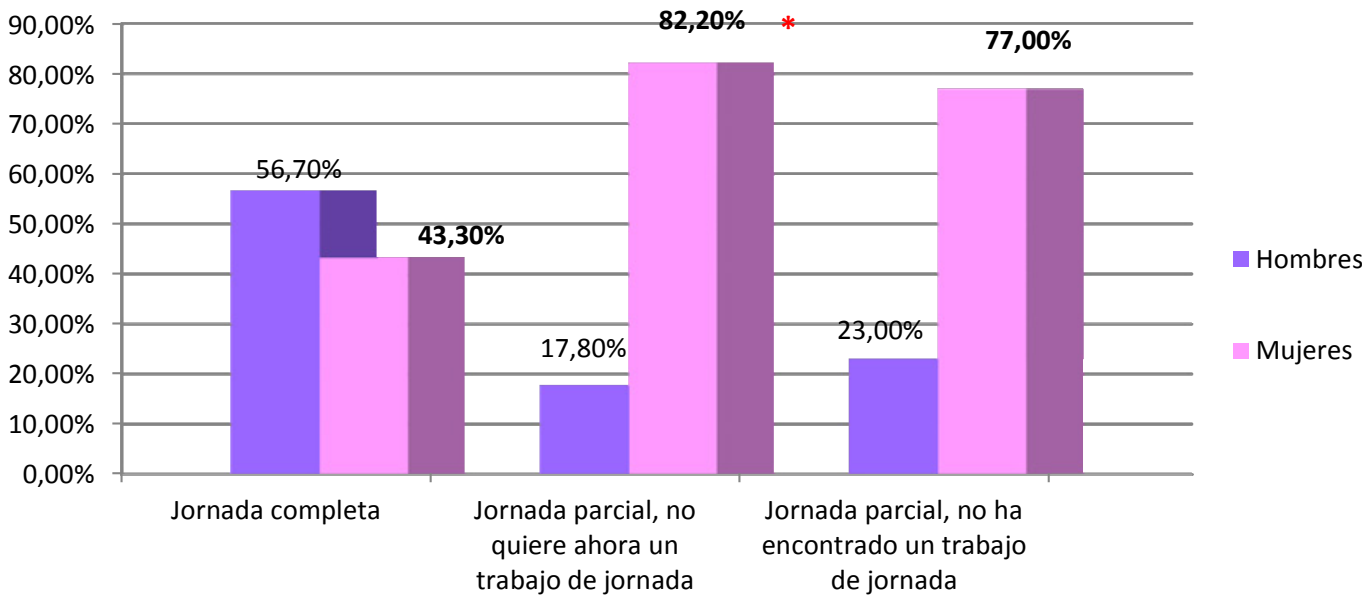

Respecto al reconocimiento médico en la pregunta sobre: si se le ha ofrecido hacerse el reconocimiento médico en los últimos 12 meses (Gráfico 4) encontramos una diferente distribución entre géneros estadísticamente significativa $(\mathrm{p}=0,05)$ con predominio de mujeres que afirmaron no haber recibido ningún ofrecimiento para hacerse el reconocimiento médico en los últimos 12 meses.

Mientras que los hombres en mayor proporción afirmaron que si se les ofreció y si se realizaron el reconocimiento médico.

Gráfico 4. Distribución de la población según género y ofrecimiento para hacerse el Reconocimiento médico

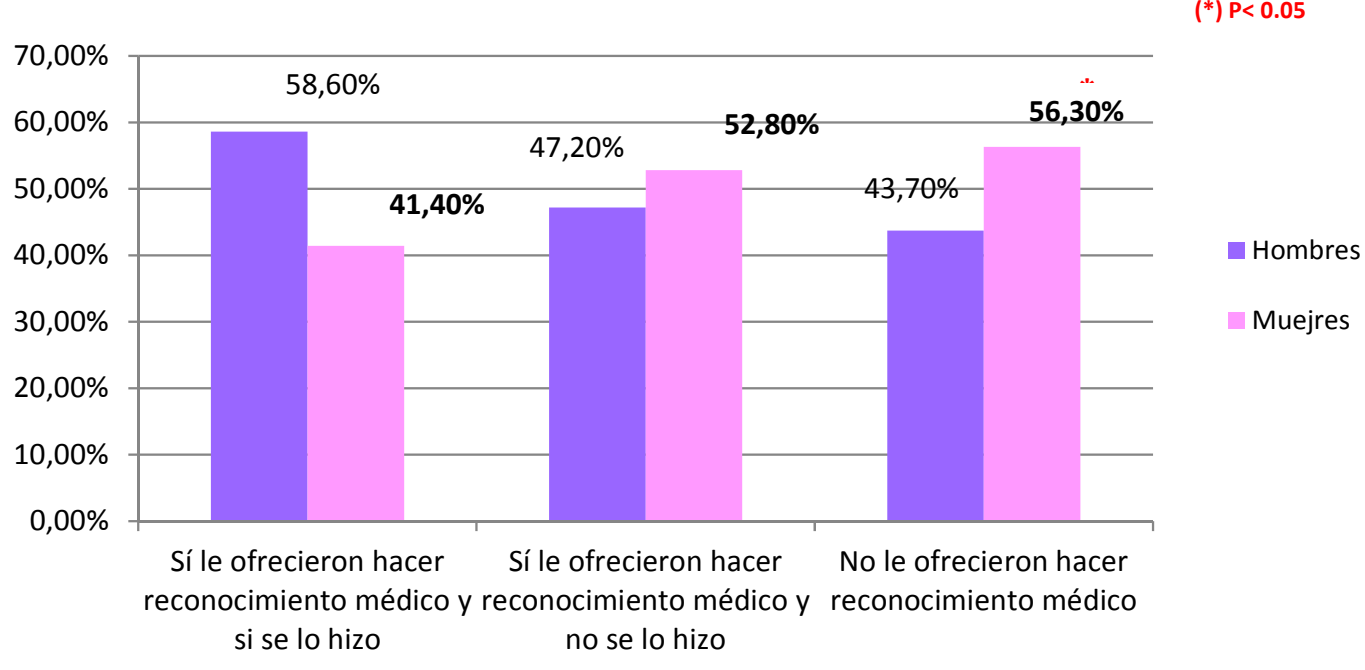

En el análisis sobre la evaluación de los riesgos para la salud en el puesto de trabajo durante los últimos 12 meses (Gráfico 5) se observa con una diferencia estadísticamente significativa $(\mathrm{p}=0,05)$ la desigualdad de género, donde la presencia de la mujer es menor en el grupo de los trabajadores a los que si se les hizo la evaluación del puesto de trabajo. Predominando igualmente la mujer, en el grupo de trabajadores a los que no se les realizo la evaluación del puesto de trabajo. 
Gráfico 5. Distribución de la población según género y evaluación de los riesgos para la salud en el puesto de trabajo durante los últimos 12 meses

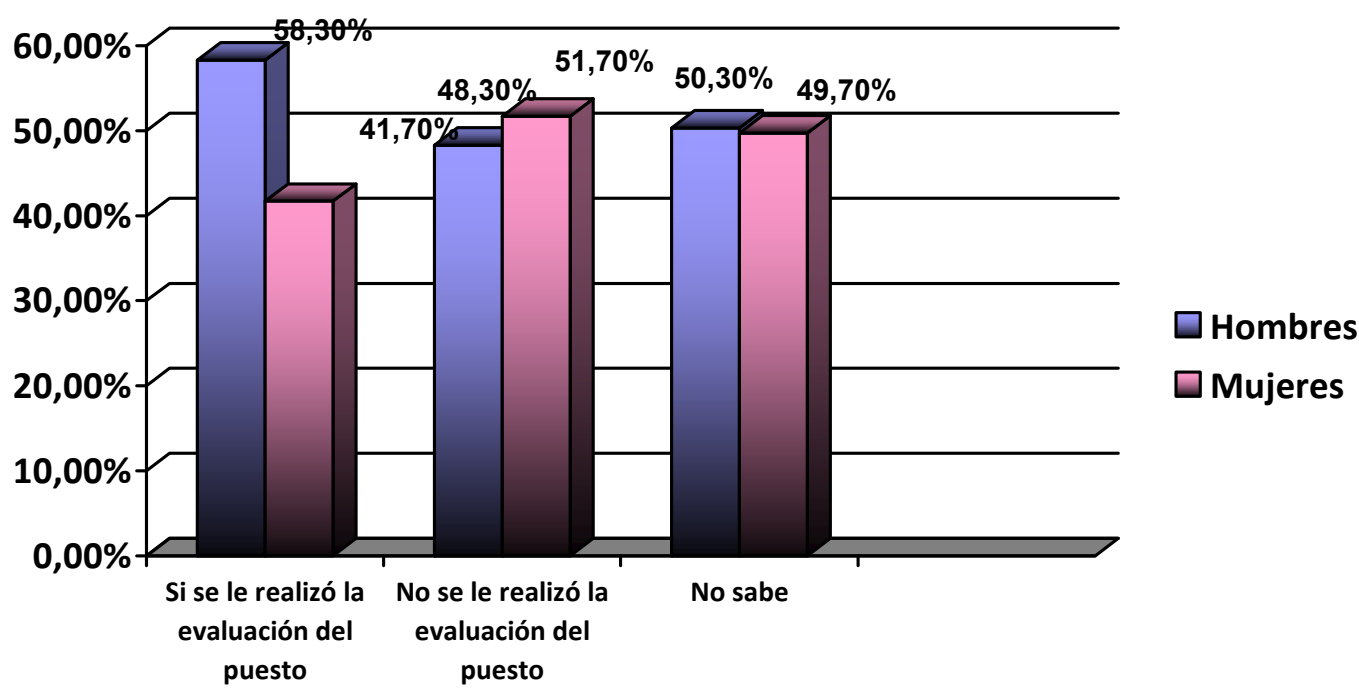

En el (Gráfico 6) sobre la evaluación de los aspectos organizativos en el puesto de trabajo, podemos observar que la mujer tiene un menor peso específico tanto en el grupo de los que afirman que si se le han estudiado, como en el grupo de los que niegan que se le hayan evaluado estos riesgos en los aspectos organizativos.

Gráfico 6. Distribución de la población según género y evaluación de los aspectos Organizativos

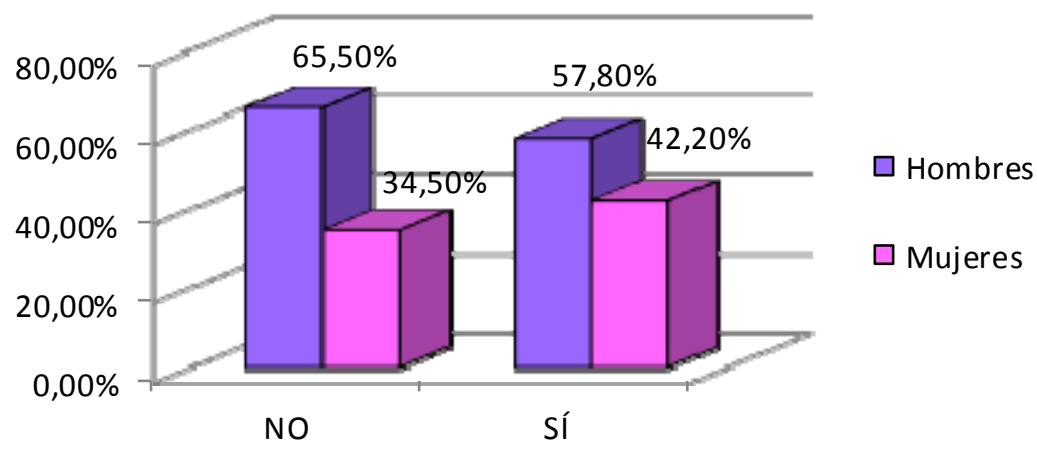

En relación al estudio de los aspectos psicosociales en el puesto de trabajo (Gráfico 7) podemos observar que, de aquellos que afirman que no se les han evaluado los riesgos psicosociales de su puesto de trabajo el género masculino tiene un mayor peso, en contraste observamos que las mujeres con una diferencia estadísticamente significativa $(\mathrm{p}=0.05)$ predominan en el grupo de los que afirma que si les evaluaron estos riesgos. 
Gráfico 7. Distribución de la población según género y evaluación los aspectos psicosociales en el puesto de trabajo

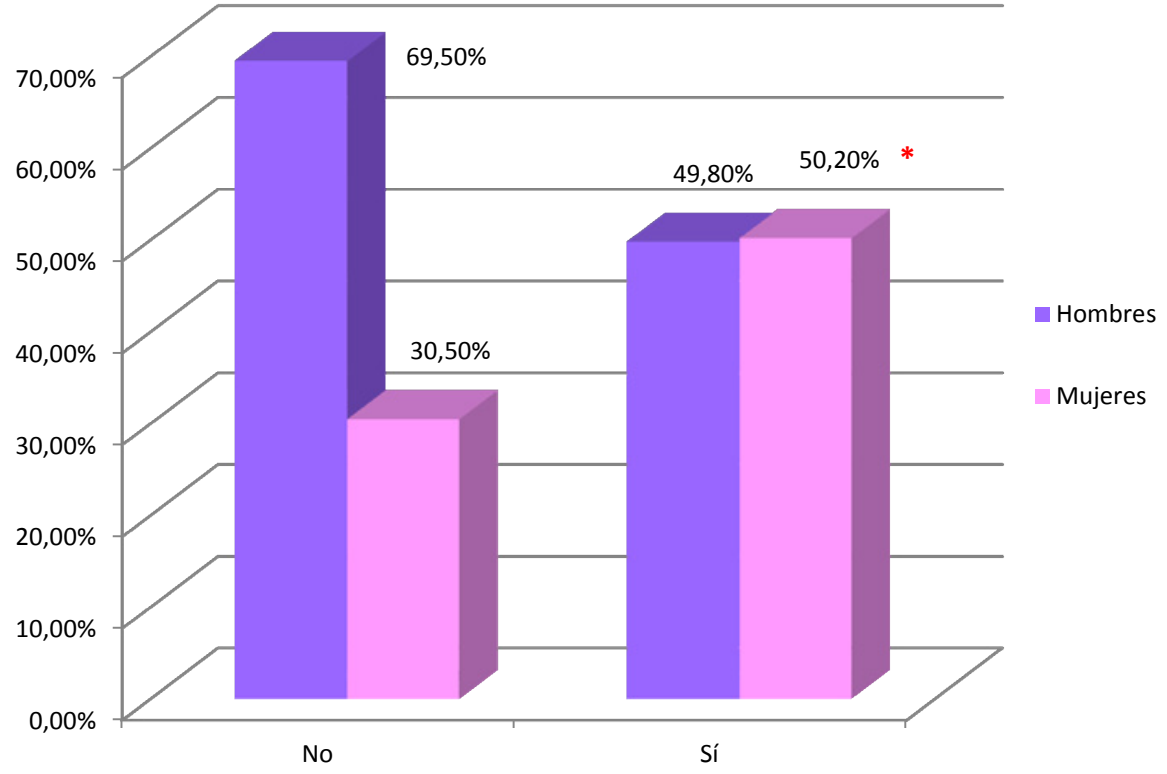

Respecto al conjunto de trabajadores que se consideran mal informados sobre los riesgos para su salud en el puesto de trabajo (Gráfico 8) observamos un claro predominio de mujeres que dicen estar mal informadas con una diferencia estadísticamente significativa $(p=0,05)$. Mientras que entre los trabajadores que dicen encontrarse bien informados, el género masculino tiene mayor peso.

Gráfico 8. Distribución de la población según género y nivel de información de los riesgos en el puesto de trabajo

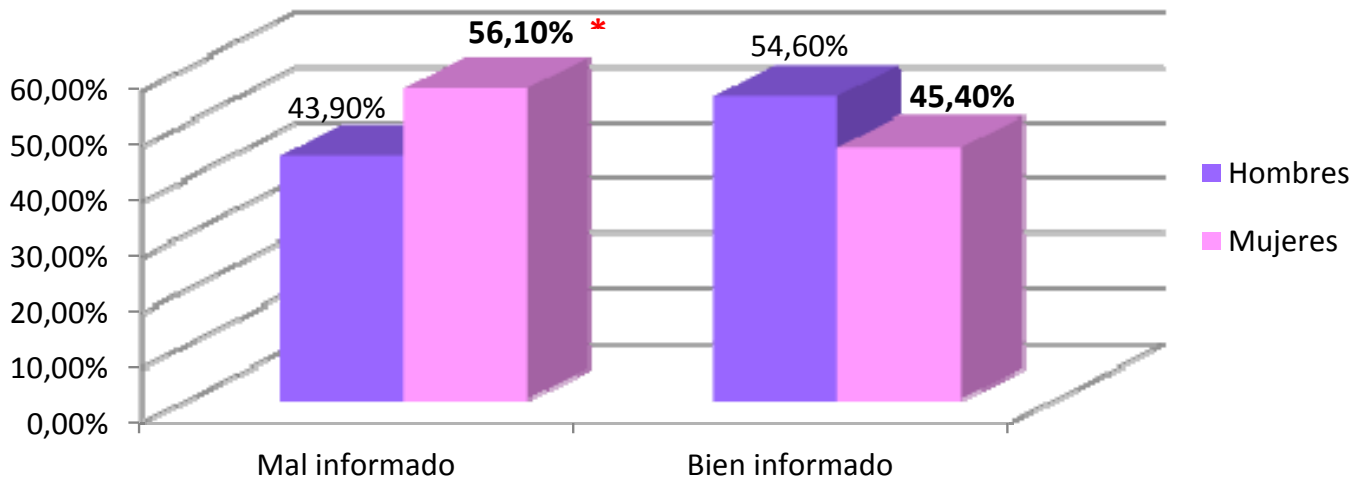

En cuanto al grupo de personas que afirmaron que sí recibieron formación sobre los riesgos en su puesto de trabajo, destaca el género masculino con un mayor peso específico. Sin embargo dentro del colectivo de trabajadores que dijeron no haber recibido formación en los últimos 12 meses, predominan las mujeres con una diferencia estadísticamente significativa. (Gráfico 9) 
Gráfico 9. Distribución de la población según género y nivel formación sobre los riesgos en el puesto de trabajo

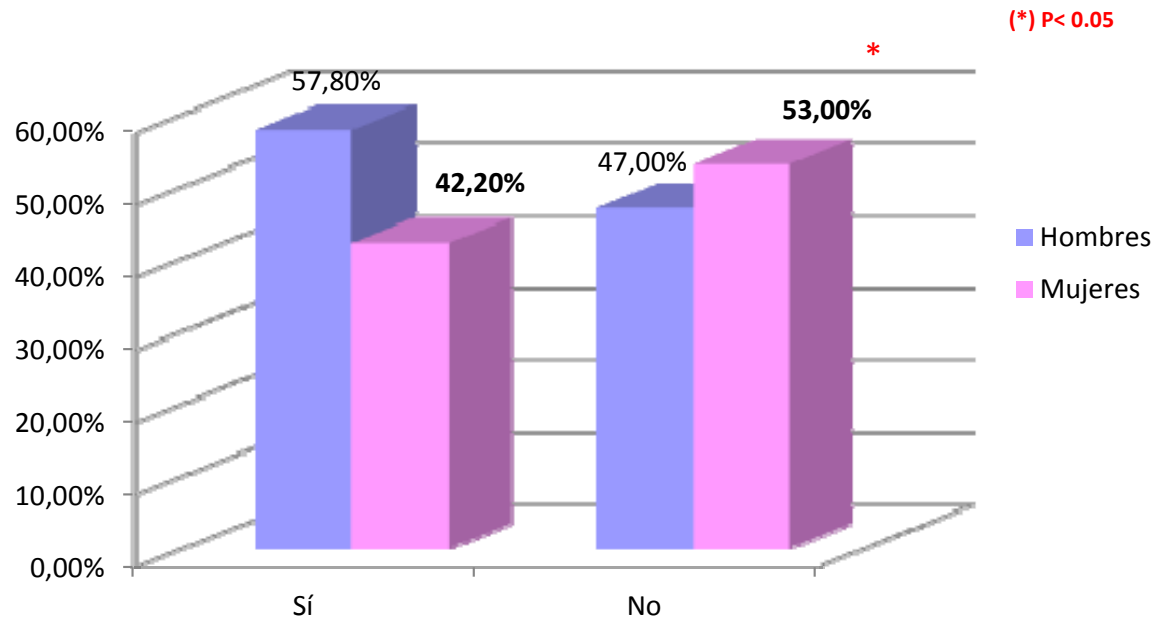

En lo referente al "ofrecimiento de reconocimiento médico» (Tabla 7), los factores que implicaron una menor oportunidad de ofrecimiento del reconocimiento médico fueron: La no existencia de delegado de prevención, el contrato temporal, ser extranjero y ser mujer. En empresas con una mayor plantilla es más frecuente que lo ofrezcan, al igual que en los sectores de la industria, y de la construcción, así como en aquellos puestos que tienen exposición a ruido.

Tabla 7. Análisis de ofrecimiento del reconocimiento médico, según género

\begin{tabular}{lccc}
\hline \multicolumn{1}{c}{ Ofrecimiento de reconocimiento médico } & OR & \multicolumn{2}{c}{ 95\% C. I } \\
\hline SEXO (mujer) & 1.418 & 1.178 & 1.707 \\
\hline NACIONALIDAD (extranjero) & 1.824 & 1.319 & 2.521 \\
\hline DELEGADO (sin delegado) & 3.207 & 2.644 & 3.890 \\
\hline TIPO DE CONTRATO (temporal) & 2.864 & 2.306 & 3.555 \\
\hline
\end{tabular}

En cuanto al haberse realizado o no el reconocimiento médico (Tabla 8), los factores que implicaron una menor oportunidad de realización del reconocimiento médico fueron: «La no existencia de delegado de prevención», "el contrato temporal» y «ser mujer».

Trabajar en los sectores de la construcción e industria se mostraron como factores asociados a un realizar más frecuentemente el reconocimiento médico.

La carga mental o el esfuerzo ergonómico no influyeron en el hecho de realizarse o no el reconocimiento médico. Los factores como tamaño de la empresa, ser extranjero, y la existencia de delegado de prevención, influyen más que el hecho de estar expuesto al riesgo.

Tabla 8. Análisis de la realización o no el reconocimiento médico, según género

\begin{tabular}{lccc}
\hline \multicolumn{1}{c}{ Haberse realizado o no el reconocimiento médico } & OR & \multicolumn{2}{c}{$95 \%$ C. I } \\
\hline SEXO (mujer) & 1.430 & 1.222 & 1.673 \\
\hline DELEGADO DE PREVENCIÓN (sin delegado) & 2.478 & 2.102 & 2.920 \\
\hline TIPO CONTRATO (temporal) & 2.156 & 1.762 & 2.640 \\
\hline
\end{tabular}

En la (Tabla 9) sobre evaluación de riesgos del puesto de trabajo, los factores que se asociaron con una menor oportunidad para la realización de la evaluación de riesgos del puesto de trabajo fueron: La no existencia de delegado de prevención, ser extranjero y tener un contrato temporal. 
Trabajar en los sectores de la construcción e industria y el nivel de estudios se asociaron con una mayor frecuencia en la evaluación de riesgos en el puesto de trabajo.

Tabla 9. Análisis de la evaluación de riesgos, según género

\begin{tabular}{lccc}
\hline \multicolumn{1}{c}{ Evaluación de riesgos } & OR & \multicolumn{2}{c}{ 95\% C. I } \\
\hline NACIONALIDAD (Extranjeros) & 1.455 & 1.050 & 2.017 \\
\hline DELEGADO DE PREVENCIÓN (Sin delegado) & 3.878 & 3.238 & 4.646 \\
\hline TIPO DE CONTRATO (Temporal) & 1.285 & 1.032 & 1.600 \\
\hline
\end{tabular}

Los factores asociados a disponer de menor información sobre los riesgos para la salud en el puesto de trabajo (Tabla 10), fueron: La no existencia de delegado de prevención, trabajar en empresas de más de 10 trabajadores, ser mujer, estar expuesto a la inhalación de sustancias químicas, riesgo ergonómico o carga mental.

Tabla 10. Análisis de la información sobre los riesgos en el puesto de trabajo, según género

\begin{tabular}{lccc}
\hline \multicolumn{1}{c}{ Información sobre riesgos } & OR & \multicolumn{2}{c}{$95 \%$ C. I } \\
\hline SEXO (Mujer) & 1.367 & 1.081 & 1.728 \\
\hline PLANTILLA - C (1) & 1.650 & 1.196 & 2.276 \\
\hline PLANTILLA - C (2) & 1.497 & 1.030 & 2.176 \\
\hline PLANTILLA - C (3) & 1.747 & 1.176 & 2.594 \\
\hline DELEGADO DE PREVENCIÓN (Sin delegado) & 3.676 & 2.892 & 4.671 \\
\hline INDUSTRIA QUÍMICA (1) (Inhalar contaminantes & 1.635 & 1.227 & 2.179 \\
químicos) & & & \\
\hline CARGA ERGONÓMICO (1) & 1.865 & 1.448 & 2.402 \\
\hline CARGA MENTAL (1) & 1.515 & 1.002 & 2.289 \\
\hline
\end{tabular}

En relación con la formación o información en prevención de riesgos laborales (Tabla 11) se repite una vez más como factor asociados a una menor oportunidad de formación e información, la no existencia de delegado de prevención, ser mujer, trabajadores extranjeros y trabajadores con contrato temporal.

Tabla 11. Análisis de la formación o información sobre los riesgos en el puesto de trabajo

\begin{tabular}{lccc}
\hline \multicolumn{1}{c}{ Formación o información } & OR & \multicolumn{2}{c}{$95 \%$ C. I } \\
\hline SEXO (Mujer) & 1.257 & 1.070 & 1.477 \\
\hline NACIONALIDAD (Extranjero) & 1.420 & 1.046 & 1.926 \\
\hline DELEGADO (Sin delegado) & 3.641 & 3.086 & 4.296 \\
\hline TIPO DE CONTRATO (Temporal) & 1.351 & 1.097 & 1.663 \\
\hline
\end{tabular}

\section{DISCUSIÓN Y CONCLUSIONES}

Por los resultados obtenidos al comparar proporciones para hacer el análisis descriptivo de los datos, encontramos una diferencia significativa de género en los aspectos preventivos analizados. Tras controlar las variables incluidas en el análisis de regresión, obtuvimos casi como una constante de factores de riesgo: el ser mujer, la ausencia del delegado de prevención, el contrato temporal, y ser trabajador extranjero.

Por otra parte, llama la atención que la vigilancia de la salud depende más de la presencia o no del delegado de prevención y del tamaño de la empresa que de los riesgos específicos de cada puesto, sugiriéndonos que no se cumple la normativa vigente la cual exige que la vigilancia de la salud debe estar en función del riesgo. 
Al comparar la gestión preventiva y el género, J. Llorca Rubio comprueba que en su aplicación existen diferencias estadísticamente significativas, en lo referente a la existencia de protección colectiva, información, formación y vigilancia de la salud, siendo el de las mujeres el colectivo más desfavorecido ${ }^{7}$.

En general observamos que el ser mujer trabajadora supuso un factor de riesgo para tener acceso a la gestión preventiva. Lo que implica un menor ofrecimiento y realización del reconocimiento médico a la mujer que a los hombres, coincidiendo con el análisis de la VI ENCT realizado por Martínez Vidal, según el cual es más probable que a las mujeres se les ofrezca en menor medida el reconocimiento médico laboral, y que también es más probable que, aunque se les haya ofrecido, éstas rehúsen hacérselo (en un 10,2\% frente al 6,4\% de los varones). Rechazo que podría tener relación con aspectos laborales (tales como horario o tipo de contrato) o con otros aspectos extra laborales relacionados con el género. De igual manera Martínez Vidal demuestra que en las actividades relacionadas con comercio, hostelería así como actividades sociales y de servicios en su mayoría ocupados por mujeres, el ofrecimiento de reconocimiento médico no llegó al 50\%, mientras que a la población que trabaja en la industria química, el reconocimiento se le ofreció al $100 \%{ }^{15}$.

M. Zimmeramn analizando la VI Encuesta Nacional de Condiciones de Trabajo encuentra que a la mayoría de los encuestados, en los últimos doce meses, se les había ofrecido, por parte de la empresa, la posibilidad de pasar un reconocimiento médico a lo que respondieron afirmativamente el $66,4 \%$ de los hombres y el 55\% de las mujeres, mostrando una diferencia estadísticamente significativas, al igual que en nuestro estudio de la VII ENCT. En lo referente a si se hizo la evaluación o análisis de riesgos en el puesto de trabajo en los últimos 12 meses, según dicho estudio respondieron afirmativamente el $36,2 \%$ de los hombres y el $29,3 \%$ de las mujeres ${ }^{10}$.

Demostrándose una vez más, las diferencias estadísticamente significativas presentes en la oferta preventiva para las actividades que ocupan predominantemente los hombres frente a las que realizan las mujeres, tal como hemos descrito en nuestro estudio.

En los resultados obtenidos de la VI Encuesta por Marta Zimmeramn, sobre el grado formación o información de los riesgos para la salud y seguridad relacionados con su trabajo. Respondieron afirmativamente el 57,9\% de los hombres y el 48,8\% de las mujeres. Es decir, que las mujeres, con una diferencia estadísticamente significativa, recibieron menor formación o información que los hombres.

En nuestro estudio de la VII encuesta ${ }^{14}$, obtuvimos igualmente un porcentaje significativamente superior de mujeres que dijeron no recibir formación e información sobre los riesgos de su puesto de trabajo, es decir que en esta materia la mujer sigue en desventaja con respecto a los hombres, desconociendo lo que la falta de formación e información sobre los riesgos y las medidas de protección puede suponer para su salud. Comprobándose una vez más, que los hombres en relación a las mujeres también reciben por parte de su empresa o su centro de trabajo una mayor consideración en estos aspectos.

Concluimos nuestro estudio corroborando la hipótesis que afirma la existencia de desigualdad de género en materia de prevención laboral. Y al alcanzar los objetivos planteados confirmamos que la mujer trabajadora muestra una significativa desventaja respecto al hombre en las actividades preventivas relacionadas con la vigilancia de la salud, control de los riesgos en el puesto de trabajo así como en formación e información. Y por todo lo expuesto podemos decir que en la actualidad todavía no se ha establecido el enfoque de género en los aspectos de salud y seguridad en el trabajo.

\section{BIBLIOGRAFÍA}

1. Guía para la incorporación de la perspectiva de género [acceso 7 Junio 2012]. Instituto de la Mujer. España. [Acceso septiembre 2014] Disponible en: SGPROGmujer@mtas.es 
2. Manual para la perspectiva de género en las políticas de empleo de inclusión social y de protección social. [Acceso 9 Junio 2012] Dirección General de Empleo, Asuntos Sociales e Igualdad de Oportunidades de la Comisión Europea.2008 [acceso septiembre 2014]. Disponible en: ec.europa.eu/social/BlobServlet? docId=2045\&langId $=$ es

3. Ley Orgánica $3 / 2007$, de 22 de marzo, para la igualdad efectiva de mujeres y hombres. BOE $\mathrm{n} .^{\circ} 71$. [Acceso septiembre 2014] Disponible en: www.boe.es

4. Bureau for Gender Equality International Labour Standars Department. [Acceso julio 2014] Gender Equality and Decent Work.2012. Disponible en: http://www.ilo.org/wcmsp5/groups/public/---ed_norm/--normes/documents/publication/wcms_088023.pdf

5. $1 .^{a}$ Conferencia mundial sobre la mujer. Confederación Sindical Internacional. [Acceso Junio 2014] Bruselas 2009. Disponible en: www.ituc-csi.org/1a-cmpm-conclusiones-y

6. Campos-Serna, J., Ronda-Pérez, E., Artazcoz, L., Benavides. Desigualdades de género en salud laboral en España. Gac. Sanit.2012 (605): 1-9 [acceso Julio 2014] Disponible en: http://www.scielosp.org/scielo. php?pid=S0213-91112012000400009\&script=sci_arttext

7. José Luis Llorca Rubio, Pedro R. Gil-Monte. Prevención de riesgos laborales y su relación con el género de los trabajadores. llorca_josrub@gva.es. Saúde Soc. São Paulo, v.22, n.3, p.727-735, 2013. [Acceso septiembre 2014] http://www.scielo.br/scielo.php?pid=S0104-12902013000300007\&script=sci_arttext

8. Roquelaure Y, Ha C, Rouillon C, Fouquet N, Leclerc A, Descatha A, Touranchet A, Goldberg M, Imbernon E. Risk factors for upper-extremity musculoskeletal disorders in the working population. 2009 Oct 15; 61(10):1425-34. [Julio 2014] Disponible en: http://onlinelibrary.wiley.com/doi/10.1002/art.24740/full

9. Valls Llobet, C. Aspectos biológicos y clínicos de las diferencias. Quarck: Ciencia, medicina, comunicación y cultura $2003 ; 27$

10. Zimmermann Verdejo, M. Mujer y trabajo: aproximación al análisis de indicadores de desigualdad a través de metodología de encuesta, Departamento de Investigación e Información. INSHT2009. [Acceso 20 Junio 2014] Disponible: www.oect.es/.../MUJER\%20Y\%20TRABAJO_INDICADORES\%20DE\%...

11. M. ${ }^{a}$ Fernanda González Gómez. Occupational health and gender. Notes to incorporate the gender mainstreaming in the occupational risks prevention field. 2011; 57. Suplemento 1: 1-262.

12. Valentina Forastieri. Women workers and gender issues on occupational safety and health-information note. [Julio 2014]. Disponible en: (http://www.ilo.org/safework/info/publications/WCMS_108003/ lang--n/index.htm

13. La mujer y la salud en el trabajo. Agencia Europea para la Seguridad y la Salud en el Trabajo. [20 Junio 2014] Disponible en: https://osha.europa.eu/es/priority_groups/gender/index_html

14. VII Encuesta Nacional de Condiciones de Trabajo. Instituto Nacional de Seguridad e Higiene del Trabajo [acceso septiembre 2014] (http://www.insht.es/).

15. Martínez Vidal, M., Álvarez Castillo, M.C., González Gómez, M.F., Sagües Cifuentes, M.J. Análisis de la VI Encuesta Nacional de Condiciones de Trabajo en la Comunidad de Madrid; 2010. Chapter 2, p. 153-35.

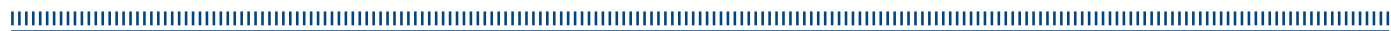

